\title{
Die Schwester der Medizin
}

\section{Piet van Spijk}

Dr. med., Dr. phil., Facharzt für Allgemeine Innere Medizin, Mitglied FMH, Präsident Forum Medizin und Philosophie

Wussten Sie, dass die Medizin eine Schwester hat? Raten Sie, wer es ist!

Nein, es ist nicht die Oekonomia, obwohl sich bei den meisten medizinischen Themen immer auch Fragen der Finanzierung und Wirtschaftlichkeit aufdrängen. Es ist auch nicht die Biologie, nicht die Physik oder die Chemie. Dies Schwester der Medizin ist die Philosophie!

\section{In letzter Zeit mehren sich die Anzeichen, dass die einseitige Ausrichtung der Medizin eine Korrektur erfährt.}

Eine der grossen grauen Eminenzen der europäischen Kulturgeschichte, Tertullian, machte das vor fast 2000 Jahren klar. Für Medizinstudierende war die Philosophie bis weit ins 19. Jahrhundert hinein ganz selbstverständlich ein Pflichtfach. Dann kam die Überzeugung auf, die Medizin gehöre zur Familie der Naturwissenschaften; das Philosophikum wurde durch das Physikum ersetzt und später durch Wahrscheinlichkeitsrechnungen und Statistik (d.h. die Mathematik) ergänzt. Die Medizin wurde zu einer naturwissenschaftlichen Disziplin, auf die Philosophie - bzw. deren
Teilwissenschaft, die Ethik - griff man vor allem dann zurück, wenn es die Anwendung umstrittener medizintechnischer Praktiken (Präimplantationsdiagnostik, Erbgutmanipulationen, passive Sterbehilfe etc.) moralisch zu rechtfertigen galt.

\section{Besinnung auf die Wurzeln}

In letzter Zeit mehren sich die Anzeichen, dass diese einseitige Ausrichtung der Medizin eine Korrektur erfährt. Als Zeichen dafür kann gewertet werden, dass gegenwärtig an drei Deutschschweizer Universitäten Nachdiplomstudien zum Thema "Philosophie und Medizin» angeboten werden. So ist es heutzutage wieder möglich, Vorteile und Nutzen eines Philosophikums kennenzulernen [1]. Andere Indizien dafür sind ein kürzlich erschienenes Buch von Beat Gerber [2] und ein Artikel von Magdalena Hoffmann in der Luzerner Standeszeitung [3]. Beide beziehen sich auf Tertullian und weisen darauf hin, dass viele medizinische Entscheidungen auch genuin philosophische Fragen enthalten. Eine kleine Auswahl philosophischer Themen in der Medizin:

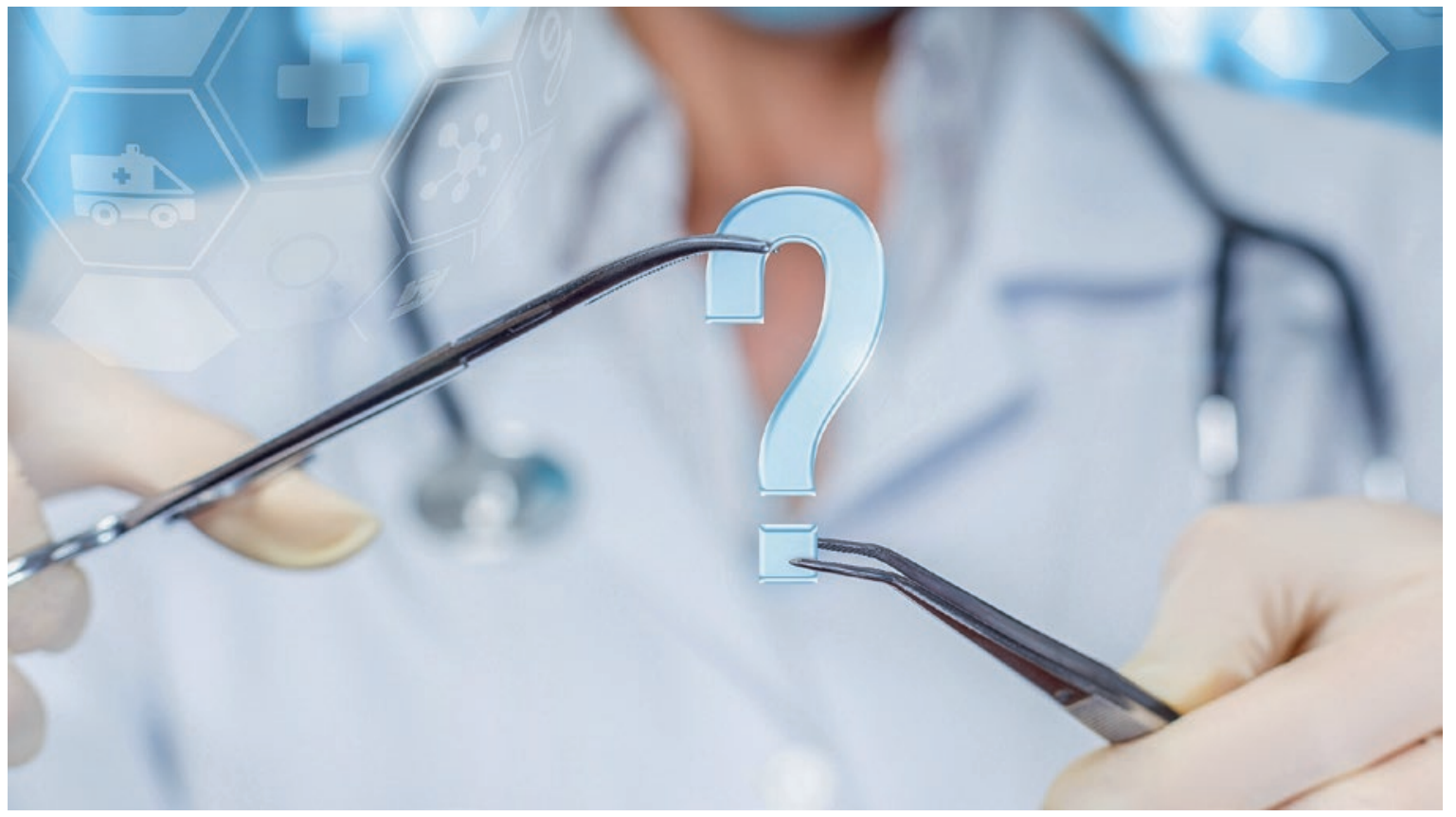

Alfred N. Whitehead: "In der philosophischen (und medizinischen) Diskussion ist die leiseste Andeutung dogmatischer Sicherheit hinsichtlich der Endgültigkeit von Behauptungen ein Zeichen von Torheit.» 
- Welchen Patientinnen und Patienten ist bei Knappheit der Ressourcen (z.B. Intensivpflege-Betten) aus welchem Grund der Vorzug zu geben?

- Ist es sinnvoll, einem 95-Jährigen eine Hüftprothese einzusetzen? Und wenn «ja», warum?

- Was ist das Ziel medizinischer Bemühungen? Ist es die Gesundheit? Was ist Gesundheit?

- Sind Viren Lebewesen? Haben sie Ziele? Sind sie intelligent?

u.a.m.

\section{Kritisches Hinterfragen}

Um nachhaltigen Antworten auf Fragen dieser Art auf die Spur zu kommen, sind solide wissenschaftstheoretische, methodologische und generell philosophische Kenntnisse unabdingbar. Es geht nicht nur darum, medizinische Probleme zu lösen, sondern auch darum, die Medizin als Wissenschaft zu reflektieren, und um ein «kritisches Hinterfragen von Gewissheiten, vertieftes Reflektieren über Bekanntes und Unbekanntes und Offenheit für Anderes und Neues» [4].

\section{Wem ist bei Knappheit der Ressourcen (z.B. Intensivpflege-Betten) aus welchem Grund der Vorzug zu geben?}

Ähnliches in der Sprache von Alfred North Whitehead, einem bedeutenden Philosophen des 20. Jahrhunderts: «In der philosophischen (und medizinischen) Diskussion ist die leiseste Andeutung dogmatischer Sicher- heit hinsichtlich der Endgültigkeit von Behauptungen ein Zeichen von Torheit» [5].

Das ist nicht alles, was die Philosophie ihrer Schwester, der Medizin, zu bieten hat. Als grösste Hilfe kann sie ihr die Geisteshaltung der Bescheidenheit nahebringen. Seit Sokrates und Platon ist für die Philosophie der

Es geht nicht nur darum, medizinische Probleme zu lösen, sondern auch darum, die Medizin als Wissenschaft zu reflektieren.

Satz «Ich weiss, dass ich nichts weiss» wegweisend. Für die Medizin kann dieser Satz nicht denselben Stellenwert einnehmen, denn er behindert durch seine geradezu verstörende Abgründigkeit das in der Medizin nötige, praktische Handeln. Gute Ärzte und Ärztinnen sind ermuntert, die Wortwahl anzupassen und sich zu sagen: "Ich weiss, wie wenig ich weiss.»

\section{Bildnachweis}

๑) Nataliia Mysik | Dreamstime.com, Symbolbild

\section{Literatur}

1 www.unilu.ch/weiterbildung/ksf/cas-philosophie-und-medizin/, www.weiterbildung.uzh.ch/programme/detail.php?angebnr=43, www.entresol.ch/lehrgaenge/lehrgang-basel/

2 Gerber B. Warum die Medizin die Philosophie braucht - Für ein umfassendes Verständnis von Krankheit und Gesundheit. Hofgrefe Verlag: Bern; 2020.

3 Hoffmann M. Die Medizin ist die Schwester der Medizin. Luzerner Arzt. 2020;122, S. 10.

4 Siehe Literaturangabe 2, S. 16

5 Whitehead AN. Prozess und Realität. 1987 (1929), S. 27 (Ergänzung in Klammer durch P. van Spijk). 